\title{
COUNTRY-WIDE HIV inCIDENCE STUdy COMPLEMENTING HIV SURVEILLANCE IN GERMANY
}

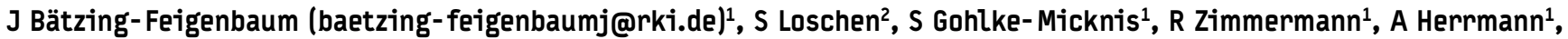 \\ 0 Kamga Wambo ${ }^{3}$, C Kücherer ${ }^{2}, 0$ Hamouda ${ }^{1}$ \\ 1. HIVIAIDS and STI Unit, Department for Infectious Disease Epidemiology, Robert Koch-Institute, Berlin, Germany \\ 2. HIV Variability and Molecular Epidemiology, Robert Koch-Institute, Berlin, Germany \\ 3. Postgraduate Training Applied Epidemiology (PAE), Department for Infectious Disease Epidemiology, Robert Koch-Institute, \\ Berlin, Germany
}

\begin{abstract}
Serological methods exist that allow differentiating between recent and long-standing infections in persons infected with HIV. During a pilot study in Berlin between 2005 and 2007 methodologies have been evaluated. In a cross-sectional study blood samples, demographic, laboratory, clinical and behavioural data based on a KABP survey were collected from patients with newly diagnosed HIV infections. The BED-CEIA was used to determine recency of infection. Recent HIV infections contributed 54\% (Cl [95\%]: 45; $64)$ in $\mathrm{MSM}$ and $16 \%(\mathrm{Cl}$ [95\%]: $0 ; 39)$ in patients with other transmission risks $(p=0.041)$. Proportions of recent infections were significantly higher in MSM $\leq 30$ years $(p=0.019)$. The mean age was 33.9 (median 34 years) in recent compared with 38.6 years (median: 38 years) in long-standing infections ( $p=0.011)$. High-risk behaviour indicated through very low condom use in recently HIV infected MSM could be identified. The results of the pilot study support expectations that the modified application of the method may contribute to improving HIV prevention efforts in Germany. On this basis the Robert Koch Institute implemented a countrywide HIV incidence study to complement HIV surveillance in early 2008. The study is funded by the German Ministry of Health. Data on recent HIV infections and current HIV transmission risks are collected. Design, methods and impact are described in detail.
\end{abstract}

\section{Background}

In Germany newly diagnosed human immunodeficiency virus (HIV) infections reached a peak of 2,360 cases in 1993. The number of cases reported to the Robert Koch Institute (RKI), the institution responsible for the national surveillance of infectious diseases in Germany, dropped continuously in the second half of the 1990s, reaching the lowest level so far in 2001 with 1,443 cases. However, since 2001 this trend has been reversed and annual case reports increased to more than 2,750 cases in 2007 [1; Figure 1]. There are several possible explanations for these changes: an increase in HIV transmission ("true" incident infections); improved (earlier) case detection and reporting following the implementation of the "Protection against Infection Act" (Infektionsschutzgesetz - IfSG) in 2001; an increased number of HIV tests performed; changing attitudes towards HIV testing; and more widespread availability of testing facilities and better access to these facilities. The limited data available suggest that the increase in HIV cases is partly due to a rising willingness to test for HIV in groups with a high risk of transmission [2]. The higher number of HIV tests (ELISA and Western blot) performed in German laboratories when comparing the year 1999 to 2004 and the augmented use of HIV-NAT in primary HIV diagnosis additionally indicate changes regarding HIV testing [3].The rising number of cases reported between 1996 and 1997 may reflect increased testing for HIV following the implementation of highly active antiretroviral treatment (HAART). Recently the upwards trend in syphilis cases reported in Germany was discussed as a possible cofactor for increased HIV transmission in men having sex with men (MSM) [1]. However, the implications of these trends have not yet been analysed systematically.

The proportion of reported HIV cases without information on the underlying transmission risk decreased from $42 \%$ to $13 \%$ between 1993 and 2007, primarily reflecting amendments concerning case reporting [1]. In the same period the proportion of cases in MSM increased from $48 \%$ to $65 \%$, whilst the proportion of cases with intravenous drug use decreased from $18 \%$ to $6 \%$. Heterosexual transmission was constant at around $15-20 \%$; persons originating from high prevalence countries (HPCs) as transmission risk for HIV contributed $11 \%$ of the total in 1993 and in 2007, with a peak of $25 \%$ in 2002 [1,2].

F I G U R E 1

Number of newly diagnosed HIV cases reported in Germany, $1993-2007(n=31,404)$

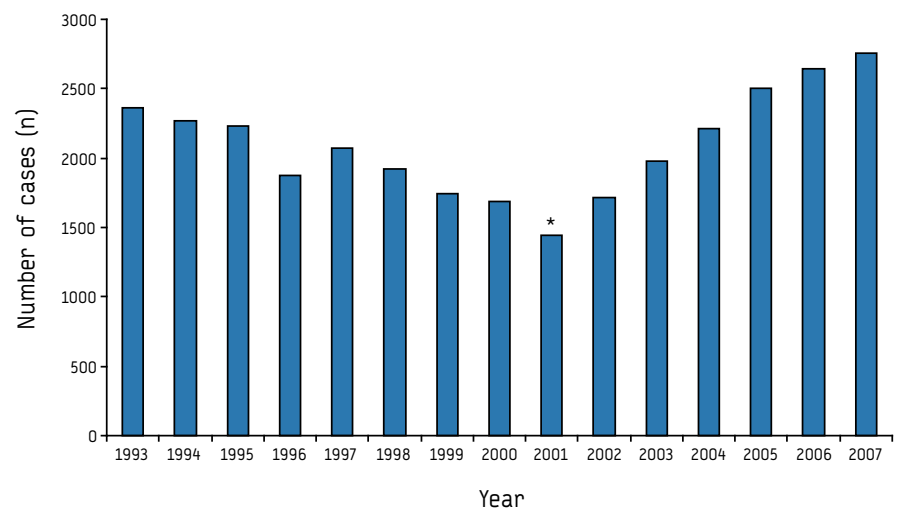

* implementation of the "Protection against Infection Act" (Infektionsschutzgesetz - IfSG) 
Standard reports of newly diagnosed HIV infections do not permit the differentiation between recently acquired (incident) and longstanding (prevalent) infections, since routinely applied serological HIV tests (screening and confirmatory tests) do not provide such information. The diagnosis of an HIV infection can be delayed by up to several years and the time between infection and diagnosis may be a number of years and vary considerably, thus estimating incidence rates accurately and effectively is difficult. However, incidence estimates are fundamental to understanding the current dynamics of the HIV epidemic.

Several other methods have proved suitable for the identification of recent (incident) HIV infections in patients with newly diagnosed HIV infections. The concept of recent infections in HIV usually covers a period up to six months prior to the diagnosis depending on the diagnostic assay used [4-9]. Testing for recent HIV infections was implemented as an additional component (anonymous and unlinked) of the national HIV surveillance systems in France [10,11], Switzerland [12] and in 22 federal states of the United States of America [13] and was used in selected population groups at risk for HIV infection in the United Kingdom and South Africa [14,15]. Collection of additional data on knowledge, attitudes, behaviour and practices (KABP survey) concerning HIV from patients identified as recently infected with HIV permits analysis of risks and protective factors effective in HIV transmission. Subpopulations at increased risk for acquiring HIV and with limited access to diagnostic services can be identified by comparing KABP data between risk groups.

After encouraging results from a pilot study in Berlin, a nationwide study including, testing for recent HIV infections and a KABP survey was started in Germany in March 2008. The study aims to provide a better picture of the current dynamics and drivers of the HIV epidemic based on incidence estimates. The results are expected to help amend the national prevention strategies.

\section{Pilot Study in Berlin 2005-2007}

A pilot study conducted in Berlin from 2005 to 2007 assessed the feasibility of the methodologies described above and the impact of the results for future HIV surveillance in Germany. The design was cross-sectional with voluntary sampling after obtaining patients' written informed consent. Sampling was anonymous and unlinked with no particular risk group being targeted. Exclusion criteria were clinical stage C HIV infection according to the US Centres for Diseases Control and Prevention (CDC) classification [16] and antiretroviral treatment. Clinicians in specialised private practices and clinic outpatient departments (OPD) collected venous blood and clinical data from adults aged 18 years or older with newly diagnosed HIV infections. Twenty of nearly 50 HIV-specialised facilities agreed to participate in the study. To determine a recent HIV infection the blood samples were tested using the BED-CEIA, one of the methods able to detect recent HIV infections serologically in patients with confirmed HIV diagnosis [17]. The BED-CEIA was established using a German HIV seroconverter sample panel with known time of seroconversion. Optimal cut-offs separating recent and long-standing samples in the reference panel were found with an optical density (ODn) of $\leq 0.8$ for the BED-CEIA and duration of infection of 20 weeks [18]. KABP data with regards to HIV/AIDS were collected through patients' questionnaires. Test results were not delivered to the patients.

\section{Results}

Of 132 cases sampled, 114 were included in the study, 18 did not meet the eligibility criteria.

The 132 cases represent $27 \%$ of all newly diagnosed HIV cases reported to the RKI from the Federal State of Berlin during the study period between November 2005 and February 2007 $(n=495)$. The total number of cases from Berlin accounted for $15 \%$ of all notifications from Germany. As far as data were available, all patients included had HIV-1 subtype B infections. Of the 114 cases meeting the eligibility criteria for the study, 102 were MSM (89\%) and 12 had other HIV transmission risks.

Proportions of recent out of newly diagnosed HIV infections were found to be $54 \%$ in MSM (95\% Confidence Interval (CI): 38-56) and $16 \%$ (95\% Cl: $32-0)$ in patients stating other risks. Proportions of recent infections were significantly higher in MSM $\leq 30$ years $(p=0.019)$, mean age was 33.9 (median 34 years) in patients with recent and 38.6 years (median: 38 years) in patients with longstanding infections $(p=0.011)$. Symptoms of acute seroconversion correlated significantly with recent HIV infections ( $p=0.009)$. Mean viral load (VL) was significantly higher in recent HIV infections compared with long-standing infections $(1,608,801 \mathrm{copies} / \mu \mathrm{l}$ and 141,951 copies/ $\mu$ l, respectively, $p=0.009$ ). A correlation was also found between recency of HIV infection and CD4 cell counts: counts $>500 / \mu \mathrm{ml}$ were indentified in recent HIV infections and counts $\leq 200 / \mu \mathrm{ml}$ in long-standing infections; however, this correlation was not statistically significant $(p=0.08)$.

Patients recruited for the pilot study showed a selection bias with samples from MSM being overrepresented (72\% MSM in all cases reported from Berlin compared with $89 \%$ in the study sample). However, comparison of basic demographic variables in case reports of MSM from Berlin and MSM in the Berlin pilot study sample did not show statistically significant differences within the study period. High-risk behaviour indicated through very low condom use in recently HIV-infected MSM could be identified: $>90 \%$ did not use condoms during sexual intercourse in the six months prior to HIV diagnosis and $19 \%$ stated that they did not use condoms despite being aware that their sexual partner had tested positive for HIV [19].

\section{Conclusions}

We were not able to produce incidence estimates since essential denominators are currently not available in Germany. Nevertheless, the results of the pilot study support expectations that the modified application of the method will contribute to amending and improving HIV prevention efforts in Germany.

National HIV Incidence Surveillance Programme 2008 - 2010

Since November 2007 the RKI initiated a nationwide study funded by the German Ministry of Health (BMG) to collect data on recent HIV infections and current HIV transmission risks. The results are expected to complement the available data on HIV from the general surveillance by identifying subpopulations presently at increased risk for acquiring HIV infections and the risks most recently having an impact on HIV transmission in Germany.

\section{Design and methods}

To obtain the desired information a cross-sectional unlinked anonymous study, with a case control component will be conducted from 1 March 2008 to 28 February 2010. Samples and data are collected over this period through either laboratories or specialised 
clinical centres. Information on screening patterns for all cases is gathered in both the laboratory and clinical study arm. As data from the two study arms cannot be linked, overlapping of sampling from patients in both study arms cannot be excluded.

\section{Laboratory study arm}

\section{Collaborating Institutions}

Newly diagnosed HIV cases in Germany are reported to the RKI by more than 200 laboratories. Only 36 labs, however, contribute significant numbers to the reporting of newly diagnosed HIV infections (significant defined as providing each at least $1 \%$ of the total number of cases reported nationally). These 36 labs are responsible for almost $70 \%$ of all reported newly diagnosed HIV cases in Germany, with the remaining approximately 170 labs reporting another 30\%. All 36 laboratories reporting high numbers of HIV infections agreed to participate in the national HIV incidence study (exhaustive sampling). Thirty-five of 51 randomly selected laboratories with HIV case reporting on a smaller scale also agreed to participate (random sample). Thus, a total of countrywide 71 laboratories will constitute the laboratory study arm.

\section{Methods}

Participating laboratories will collect plasma or serum samples from all newly diagnosed HIV cases during the study period. Samples are provided as "Dried Plasma Spots" (DPS) or "Dried Serum Spots" [20] and sent every month to the project group HIV Variability and Molecular Epidemiology at the RKI. All samples are tested for recency of HIV infection using the BED-CEIA. Clinical data are limited to information reported according to the national HIV surveillance regulations [21]. Data will allow to estimate recent HIV infections and incidence proportions by using basic demographic data and to analyse the risks to acquire an HIV infection. Data collected in this study arm are expected to be representative for Germany. The sample size is expected to include 1,600 cases annually representing around $60 \%$ of all new HIV diagnoses.

\section{Clinical study arm}

\section{Collaborating Institutions}

Over 80 clinical facilities specialised in HIV diagnosis and care from six regions in Germany will participate in the clinical study arm. The regions selected include those reporting the highest HIV case numbers nationally since 2001 (Figure 2) and they are characterised by a concentration of medical facilities specialised in HIV care compared with other regions. These facilities include private practitioners, clinic OPDs and counselling centres run by local health authorities or non-government organisations (NGO).

\section{Methods}

In this study arm clinicians specialised in HIV diagnosis and care will recruit patients with newly diagnosed HIV infections (cases) and patients undergoing an HIV test with negative result (controls). Cases and controls will be matched by basic demographic variables and their risk of HIV transmission. HIV testing for cases and their respective controls has to be performed within a three month period. After obtaining written informed consent, blood samples are collected from case patients as DBS [22]. The samples are analysed for recency of HIV infection by BED-CEIA at the HIV Variability and Molecular Epidemiology project group of the Robert Koch Institute. Clinical and medical history data from case and control patients are collected through a physician's questionnaire. KABP-data are collected from cases and controls by using a selfadministered patient's questionnaire. The expected sample size is 600 cases and controls annually. Analyses of the data will allow comparison between patients with recently acquired HIV infection and persons undergoing HIV tests with a negative test result in the same clinical institutions and in an identical time frame. The analyses aim at obtaining information on the current status of general knowledge about HIV/AIDS, on the behaviour and attitudes towards prevention of HIV transmission, and on the risks taken with regards to HIV transmission.

\section{Impact}

The study offers an outstanding opportunity to identify recent HIV infections out of newly diagnosed cases and estimate HIV incidence. As a result of this a deeper insight into the transmission dynamics of the ongoing HIV epidemic in Germany will be available. To prevent further HIV infections, comparative analyses are aimed at identifying the risks for HIV transmission and the relevant behaviour and attitudes. However, the major limitations of our study are insufficient screening patterns that only reflect those patients requesting an HIV test. True incidence estimates will be

F I G U R E 2

Cumulative incidence of newly diagnosed cases of HIV in Germany, 2001-2006 and six regions of the clinical study arm, Germany 2008

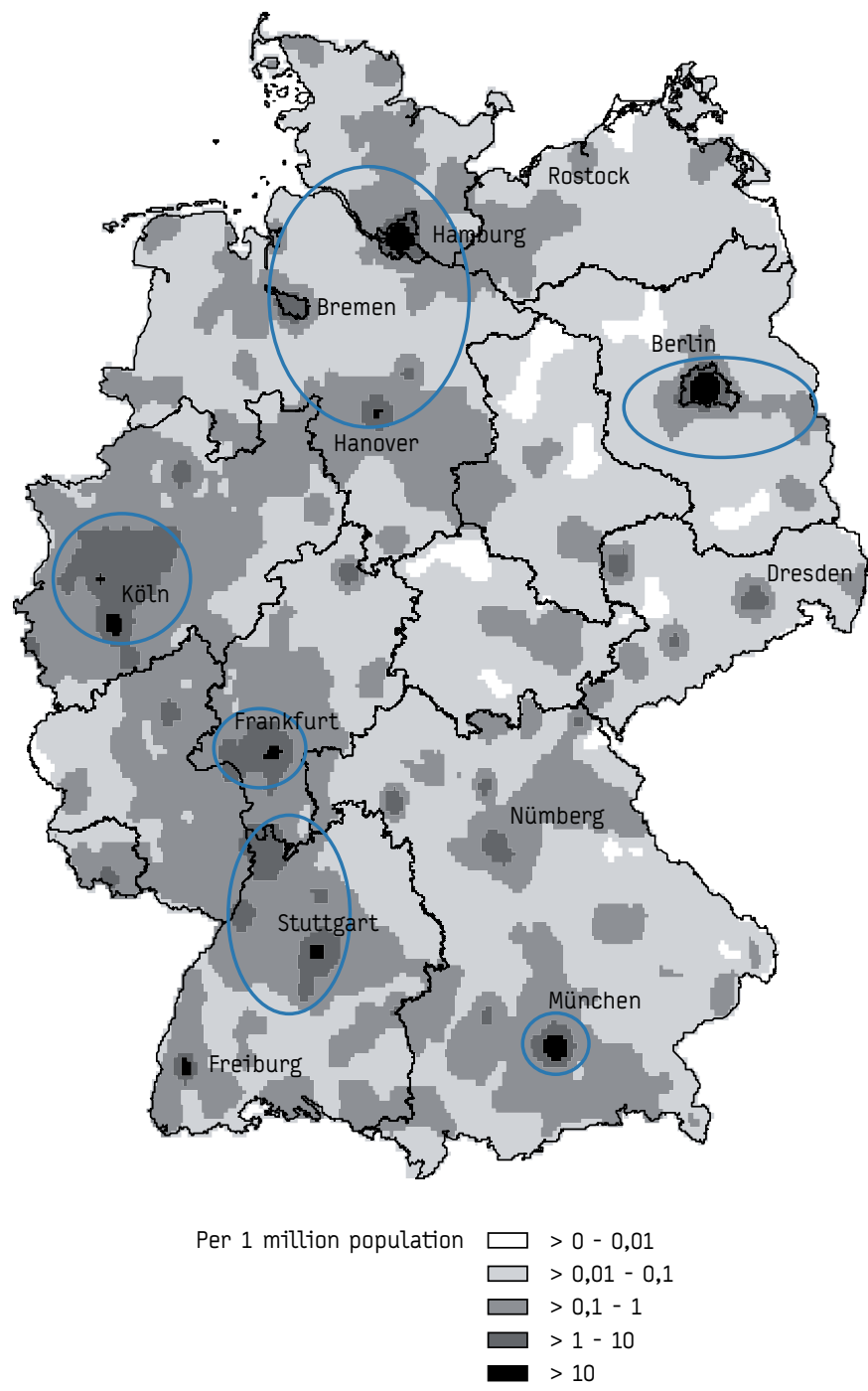


difficult to obtain as the denominators needed are not available in Germany. Despite these limitations the data are expected to have an impact on amending and improving national prevention efforts and strategies in Germany. Better knowledge of the factors driving the HIV epidemic and of the most recent dynamics of the epidemic revealing subgroups currently at increased risk of acquiring HIV will help to design targeted and prompt interventions.

\section{Acknowledgements}

The authors would like to thank all collaborating laboratories and clinical centres participating in this study for their excellent work and support.

\section{References}

1. Hamouda 0, Marcus U. HIV infections and AIDS cases in Germany. Current epidemiological data (as of 1 March 2008). [In German]. Epid Bull. A/2008:1 16(special issue). Available from: http://www.rki.de/cln_091/nn_208946/DE/ Content/Infekt/EpidBull/Archiv/2008/Sonderausgaben/A__08, templateId=raw ,property=publicationFile.pdf/A_08.pdf

2. Hamouda 0 , Marcus U. HIV infections and AIDS cases in Germany. Current epidemiological data (as of 1 March 2007). [In German]. Epid Bull. A/2007:14(special issue). Available from: http://www.rki.de/cln_091/nn_208946/DE/ Content/Infekt/EpidBull/Archiv/2007/Sonderausgaben/A__2007, templateId=r aw,property=publicationFile.pdf/A_2007.pdf

3. Wiese-Posselt M, Voss L. HIV screening and confirmatory tests in Germany in the year 2004. [In German]. Epid Bull. 2006;1:2-5. Available from: http://www. rki.de/cln_091/nn_208946/DE/Content/Infekt/EpidBull/Archiv/2006/01_06, te mplateId=raw, property=publicationFile.pdf/01_06.pdf

4. Barin F, Meyer L, Lancar R, Deveau C, Gharib M, Laporte A, et al. Development and validation of an immunoassay for identification of recent human immunodeficiency virus type 1 infections and its use on dried serum spots. J Clin Microbiol. 2005:43(9):4441-7.

5. Dobbs T, Kennedy S, Pau CP, McDougal JS, Parekh BS. Performance characteristics of the immunoglobulin G-capture BED-enzyme immunoassay, an assay to detect recent human immunodeficiency virus type 1seroconversion. J Clin Microbiol. 2004:42(60:2623-8.

6. Janssen RS, Satten GA, Stramer SL, Rawal BD, O'Brien TR, Weiblen BJ, et al. New testing strategy to detect early HIV-1 infection for use in incidence estimates and for clinical and prevention purposes. JAMA. 1998;280(1):42-8.

7. McDougal JS, Parekh BS, Peterson ML, Branson BM, Dobbs T, Ackers M, et al. Comparison of HIV type 1 incidence observed during longitudinal follow-up with incidence estimated by cross-sectional analysis using the BED capture enzyme immunoassay. AIDS Res Hum Retroviruses. 2006;22(10):945-52.

8. Parekh BS, Kennedy MS, Dobbs T, Pau CP, Byers R, Green T, et al, Quantitative detection of increasing HIV type 1 antibodies after seroconversion: a simple assay for detecting recent HIV infection and estimating incidence. AIDS Res Hum Retroviruses. 2002;18(4):295-307.

9. Suligoi B, Massi M, Galli C, Sciandra M, Di Sora F, Pezzotti P, et al. Identifying recent HIV infections using the avidity index and an automated enzyme immunoassay. J Acquir Immune Defic Syndr. 2003;32(4);424-8.

10. Lot F, Semaille C, Cazerin F, Barin F, Pinget R, Pillonel J, et al. Preliminary results from the new HIV surveillance system in France. Euro Surveill. 2004;9(10):34-7. Available from: http://www.eurosurveillance.org/em/ v09n10/0910-224.asp

11. Semaille C, Barin F, Cazein F, Pillonel J, Lot F, Brand D, et al. Monitoring the Dynamics of the HIV Epidemic Using Assays for recent Infection and serotyping among New HIV Diagnoses: Experience after 2 Years in France. J Inf Dis. 2007;196(3):377-83.

12. Schüpbach J, Gebhardt MD, Tomasik Z, Niederhauser C, Yerly S, Bürgisser P, et al. Assessment of recent HIV-1 infection by a line immunoassay for HIV-1/2 confirmation. PLoS Med. 2007;4(12):e343.

13. Hall HI, Song R, Rhodes P, Prejean J, An Q, Lee LM, et al. Estimation of HIV incidence in the United states. JAMA. 2008;300(5): 520-9.

14. Fisher M, Pao D, Murphy G, Dean G, McElborough D, Homer G, et al. Serological testing algorithm shows rising HIV incidence in a UK cohort of men having sex with men: 10 years application. AIDS. 2007;21(17):2309-14.

15. Rehle T, Shisana O, Pillay V, Zuma K, Puren A, Parker W. National HIV incidence measures - new insights into the South African epidemic. S Afr Med J. 2007;97(3):194-9.
16. Centers for Disease Control and Prevention. 1993 revised classification system for HIV infection and expanded surveillance case definition for AIDS among adolescents and adults. MMWR Recomm Rep. 1992:41(RR-17):1-19. Available from: www.cdc.gov/mmwr/preview/mmwrhtml/00018871.htm

17. Parekh BS, McDougal JS. Application of laboratory methods for estimation of HIV-1 incidence. Indian J Med Res. 2005;121(4):510-8.

18. Loschen S, Bätzing-Feigenbaum J, Poggensee G, Cordes C, Hintsche B, Rausch M, et al. Identification of recent HIV-infection: Comparison of the HIV-1 specific IgG capture ELISA (BED-CEIA) with the avidity index method. J Clin Microbiol. 2008;46(1):341-5.

19. Bätzing-Feigenbaum J. Estimating incidence of HIV infections in Germany Results from a pilot study and perspectives for HIV surveillance. [In German]. Epid Bull. 2008;1:1-4. Available from: http://www.rki.de/cln_091/nn_195972/DE/ Content/Infekt/EpidBull/Archiv/2008/01_08, templateId=raw,property=public ationFile.pdf/01_08.pdf

20. Loschen S, Bätzing-Feigenbaum J, Gohlke-Micknis S, Poggensee G, Jansen A, Hamouda 0 , et al. Differentiation of recent and chronic HIV-infections from filter dried-plasma-spots. Eur J Med Research. 2007; 12 (Suppl II):118.

21. Hamouda 0. HIV/AIDS surveillance in Germany. J Acquir Immune Defic Syndr. 2003;32 (Suppl I):S49-54.

22. Lukacs Z, Dietrich A, Ganschow R, Kohlschütter A, Kruithof R. Simultaneous determination of HIV antibodies, hepatitis C antibodies, and hepatitis B antigens in dried blood spots - a feasibility study using a multi-analyte immunoassay. Clin Chem Lab Med. 2005;43(2):141-5.

This article was published on 4 September 2008.

Citation style for this article: Bätzing-Feigenbaum J, Loschen S, Gohlke-Micknis S, Zimmermann R, Herrmann A, Kamga Wambo O, Kücherer C, Hamouda O. Country-wide HIV incidence study complementing HIV surveillance in Germany. Euro Surveill. 2008;13(36):pij=18971. Available online: http://www.eurosurveillance.org/ViewArticle. aspx?ArticleId $=18971$ 